\title{
EDITORIAL
}

\section{Microdischarges for analytical applications}

\author{
Joachim Franzke
}

Published online: 10 August 2009

(C) Springer-Verlag 2009

The first miniaturized discharges used for analytical applications were investigated nearly two decades ago to get less expensive instruments and smaller, more portable instrumentation for optical spectrometry. Direct current and high frequency induced microplasmas have been used as excitation and ionization sources for optical emission spectrometry as well as for absorption spectrometry and mass spectrometry, respectively. These plasmas have to dissociate the molecules that are introduced and the resulting atoms have to be excited or even ionized.

Experimental data such as the dimensions of the discharge, the resulting volume, the pressure, the input power, the flow rate, the detected analyte and the limit of detection are important parameters for the development of a miniaturized discharge for optical spectrometry. The mean power density and the gas exchange rate can be calculated from these experimental data. For elemental analysis, the mean power density, which is the ratio of the input power and the volume of a discharge, must be high enough to dissociate and to excite molecules, without causing damage.

I hope the following compilation of articles will give some insight into the application of microdischarge plasmas, not only in atomic spectrometry, but also in analytical and bioanalytical chemistry.

J. Franzke $(\bowtie)$

ISAS - Institute for Analytical Sciences,

Bunsen-Kirchhoff-Str. 11,

44139 Dortmund, Germany

e-mail: franzke@isas.de
Microdischarges are applied in a variety of sensing applications for chemical sensing in gas-phase environments and liquid-phase environments. Other developments analyse liquid samples by introducing them into the microplasma device using an electrothermal, mini in-torch vaporization sample introduction system. Furthermore, microdischarges can be applied to sense pressure in hightemperature environments as well as for wireless signalling radiation sensors.

The miniaturization of discharges generates different kinds of discharges that are applied for a variety of analytical excitation and ionization purposes in analytical chemistry. In comparison with the known analytical discharges, different dielectric barrier discharges are characterized by their application fields for analytical chemistry.

In the last few years, low-temperature microplasmas have become more and more interesting as a source for soft ionization. Low-temperature plasmas are explored as an ion source for the rapid screening of pharmaceutical samples with organic mass spectrometry. This gives the opportunity for high-throughput sampling for rapid screening of active ingredients in pharmaceuticals research without any sample pretreatment. This could be potentially applied as a useful tool for drug quality control.

Low-temperature plasmas can also be used for plasma stencilling for the regioselective oxidation of polydimethysiloxane and polystyrene substrates to generate digital hydrophilic-hydrophobic patterns. These substrates can be used for highly effective and long-term cell patterning of different cell lines, including human keratinocytes and embryonic kidney cells. 
Although most of the plasmas are diminished drastically in size, with the advantage of being operated at atmospheric pressure and therefore making the use of a bulky vacuum pump unnecessary, the generators are as big as before. Therefore, electrical battery driven generators with smaller dimensions have to be developed for microplasmas.

It is desirable that the contributions will stimulate further research in the field of characterization and development of microdischarge sources. Hopefully, plasma discharges will be used in further application fields of analytical and bioanalytical chemistry.

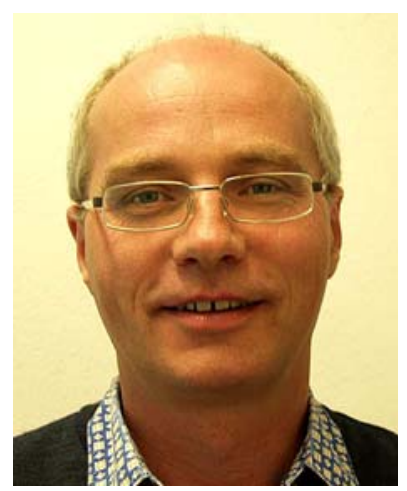

Joachim Franzke is Director of the Department of Miniaturization at ISAS. His research interests are related to the development of methods and miniaturized instrumentation for discharge-based analytical spectrometry. 